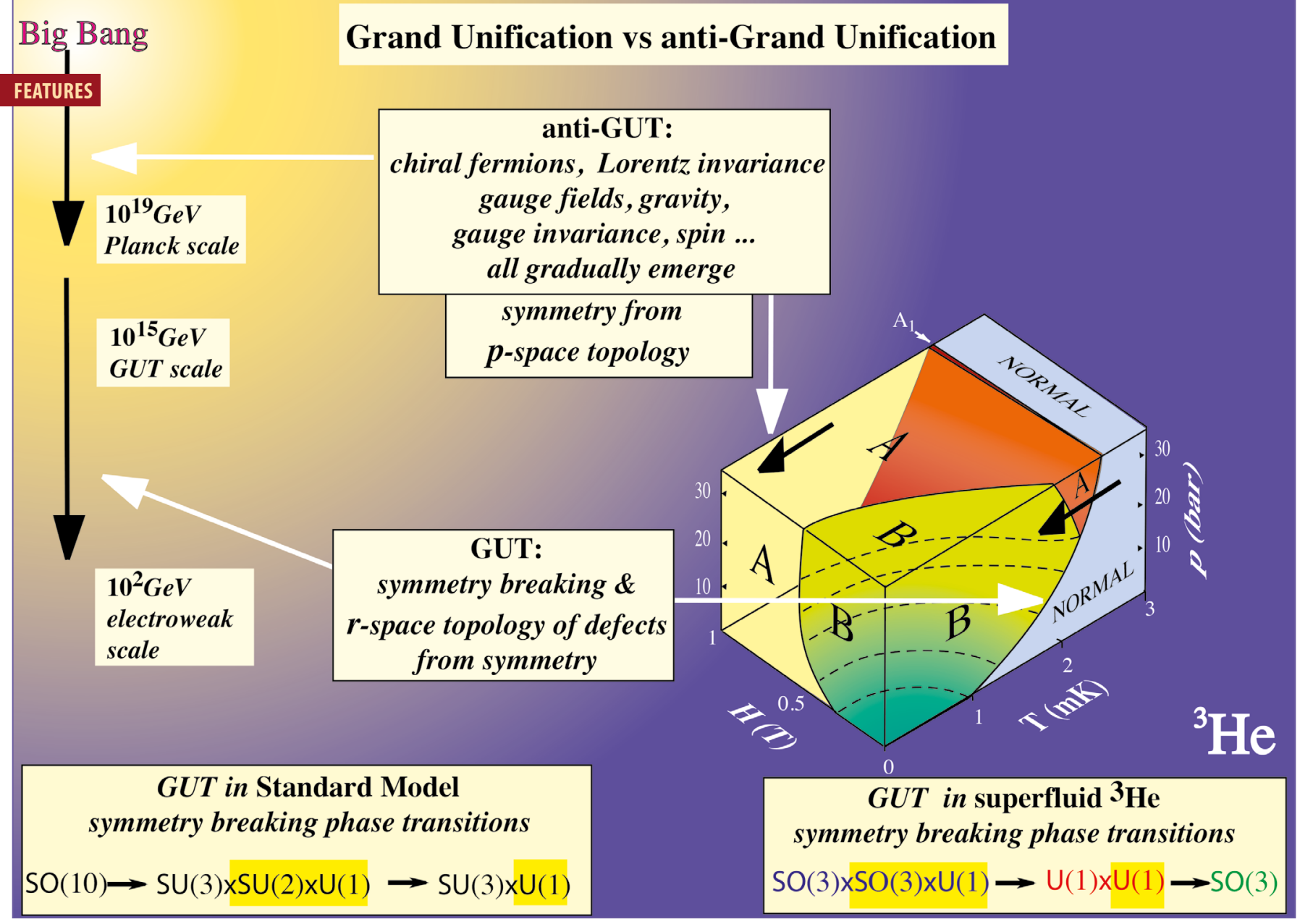

\title{
LESSONS FROM TOPOLOGICAL SUPERFLUIDS: SAFE AND DANGEROUS ROUTES TO ANTISPACETIME
}

ע.B. Eltsov ${ }^{1}$, J. Nissinen ${ }^{1}$ and G.E. Volovik ${ }^{1,2}$ - DOI: https://doi.org/10.1051/epn/2019504

- ${ }^{1}$ Low Temperature Laboratory, Aalto University, P.O. Box 15100, FI-00076 Aalto, Finland

n ${ }^{2}$ Landau Institute for Theoretical Physics, acad. Semyonov av., 1a, 142432, Chernogolovka, Russia

\section{All realistic second order phase transitions are undergone at finite transition rate and} are therefore non-adiabatic. In symmetry-breaking phase transitions the non-adiabatic processes, as predicted by Kibble and Zurek [1, 2], lead to the formation of topological defects (the so-called Kibble-Zurek mechanism). The exact nature of the resulting defects depends on the detailed symmetry-breaking pattern.

or example, our universe - the largest condensed matter system known to us - has undergone several symmetry-breaking phase transitions after the Big Bang. As a consequence, a variety of topological defects might have formed during the early evolution of the Universe. Depending on the Grand Unified Theory model, a number of diffierent cosmic topological defects have been predicted to exist. Among them are point defects, such as the 't Hooft-Polyakov magnetic monopole [3, 4], linear defects known as cosmic strings [1], surface defects or cosmic domain walls, continuous topological and nontopological objects (skyrmions and Q-balls), etc.

The model predictions can be tested in particle accelerators (now probing energy densities $>10^{-12} \mathrm{~s}$ after the Big Bang) and in cosmological observations (which have not yet identified such defects to date). The same physics, however, can be probed in symmetry breaking transitions in condensed matter systems - in fermionic superfluid ${ }^{3} \mathrm{He}$ to an astonishing degree of similarity.

The physics of Kibble-Zurek formation of cosmic string defects during a second order phase transition was 
tested in superfluid ${ }^{3} \mathrm{He}$ in a rotating cryostat (Fig. 1). With neutron irradiation local mini Big-Bangs - hot spots with temperature above the superfluid transition - were created [5]. Cooling down back into the superfluid state produced topological defects - quantized vortices - in agreement with detailed theoretical predictions.

\section{Superfluid ${ }^{3} \mathrm{He}$ as an analog of the fermionic quantum vacuum}

This is but one of the many connections of the superfluid phases of liquid ${ }^{3} \mathrm{He}$ with particle physics and general relativity [6]. All superfluid phases of ${ }^{3} \mathrm{He}$ are examples of topological superfluids with emergent relativistic excitations and topological defects.

1) In the A-phase of superfluid ${ }^{3} \mathrm{He}\left({ }^{3} \mathrm{He}-\mathrm{A}\right)$ the chiral mirror symmetry is spontaneously broken, as in the vacuum of Standard Model, where the behaviour ofleft-handed and right-handed elementary fermions (e.g. quarks and leptons) is essentially different. The fermionic excitations of ${ }^{3} \mathrm{He}-\mathrm{A}$ (called quasiparticles and -holes) are very similar to elementary particles (and antiparticles) in the early Universe, where quarks and leptons were still massless. Quasiparticles have the relativistic energy spectrum, $E^{2}=g^{i k} p_{i} p_{k}$, where the anisotropy tensor $g^{i k}$ plays the role of metric tensor in general relativity. These quasiparticles have a quantum "spin" parallel (Fig. 2 a) or antiparallel to their direction of momentum - giving the particles a handedness. The chiral right-handed particles "spin" anticlockwise whereas the left-handed clockwise (and vice versa for the chiral antiparticles).

They move in the synthetic gravitational and electromagnetic fields created by the deformations of the superfluid. Their motion is governed by an equation of the form of the relativistic Weyl equation - the linear Dirac equation applied to chiral particles. In words, the Weyl fermions of ${ }^{3} \mathrm{He}-\mathrm{A}$ experience the same quantum effects as the elementary fermions of our Universe. Strikingly they can be created and annihilated from the quantum vacuum not only as particle-antiparticle

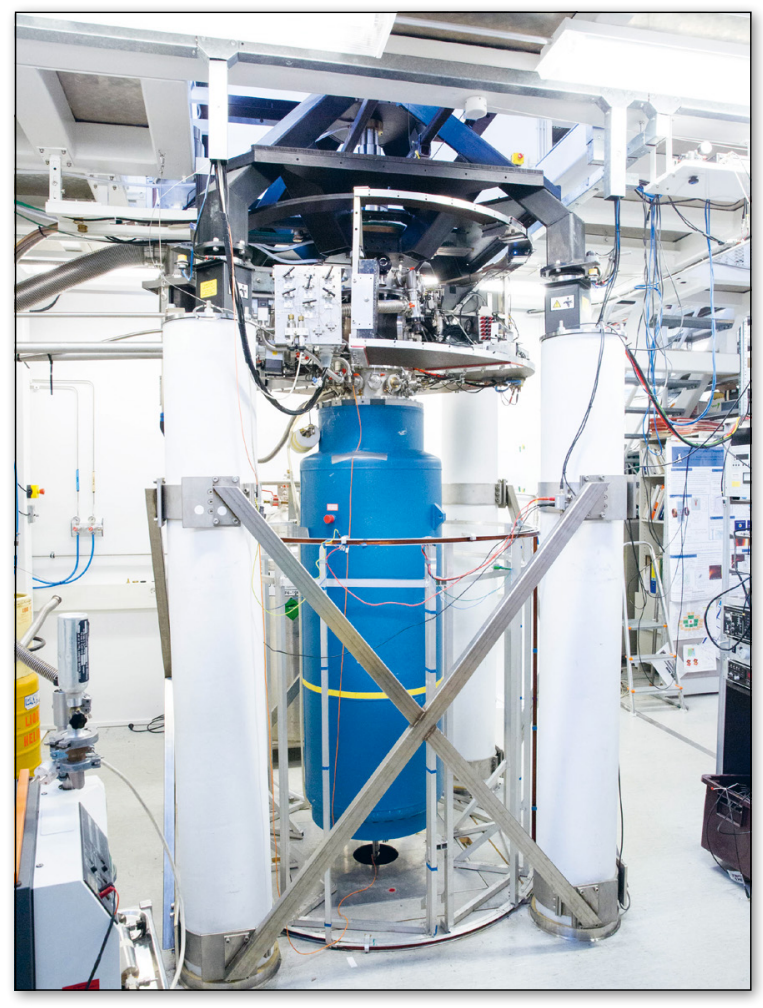

4FIG. 1: Rotating cryostat, where topological phases of superfluid ${ }^{3} \mathrm{He}$ are studied at ultralow temperature reaching $140 \mu \mathrm{K}$. Rotation allows to create and stabilize different types of topological objects, such as Alice strings, Witten superconducting strings, KibbleLazarides-Shafi walls bounded by strings, solitons, skyrmions, etc., and perform "cosmological" experiments, such as simulation of the Kibble-Zurek mechanism of defect formation in early Universe, and transition from Minkowski to Euclidean spacetime.

pairs (quasiparticles and -holes), but one by one out of vacuum fluctuations, if axial "electric" and "magnetic" fields are applied. The quantum effect of creation of chiral particles from the vacuum fluctuations is known in particles physics as the chiral or axial anomaly. In ${ }^{3} \mathrm{He}-\mathrm{A}$ the chiral anomaly has been demonstrated in experiments with skyrmions [7].

The reason for such a close and robust connection between ${ }^{3} \mathrm{He}$-A and the quantum vacuum of Standard Model is topological. The quantum vacuum of the early Universe and ${ }^{3} \mathrm{He}-\mathrm{A}$ belong to the same universality class of topological materials. This is the class of fermionic vacua with Weyl points - topologically protected points in momentum space, where the energy of a (quasi)particle goes to zero. The topology of this point in momentum space is similar to the topology of

VFIG. 2: Topological materials and fermionic quantum vacua as topologically stable configurations in momentum space. (a) Weyl point in ${ }^{3} \mathrm{He}-\mathrm{A}$ as monopole in p-space. The hedgehog of spins in momentum space is responsible for the topological stability of elementary particles of Standard Model and Weyl quasiparticles in ${ }^{3} \mathrm{He}-\mathrm{A}$. (b) The polar phase of ${ }^{3} \mathrm{He}$ has the Dirac nodal line in the quasiparticles spectrum — the p-space counterpart of a cosmic string in real space. (c) Skyrmion configurations in p-space describe the topological insulators and the fully gapped topological superfluids, such as ${ }^{3} \mathrm{He}-\mathrm{B}$.He-A.

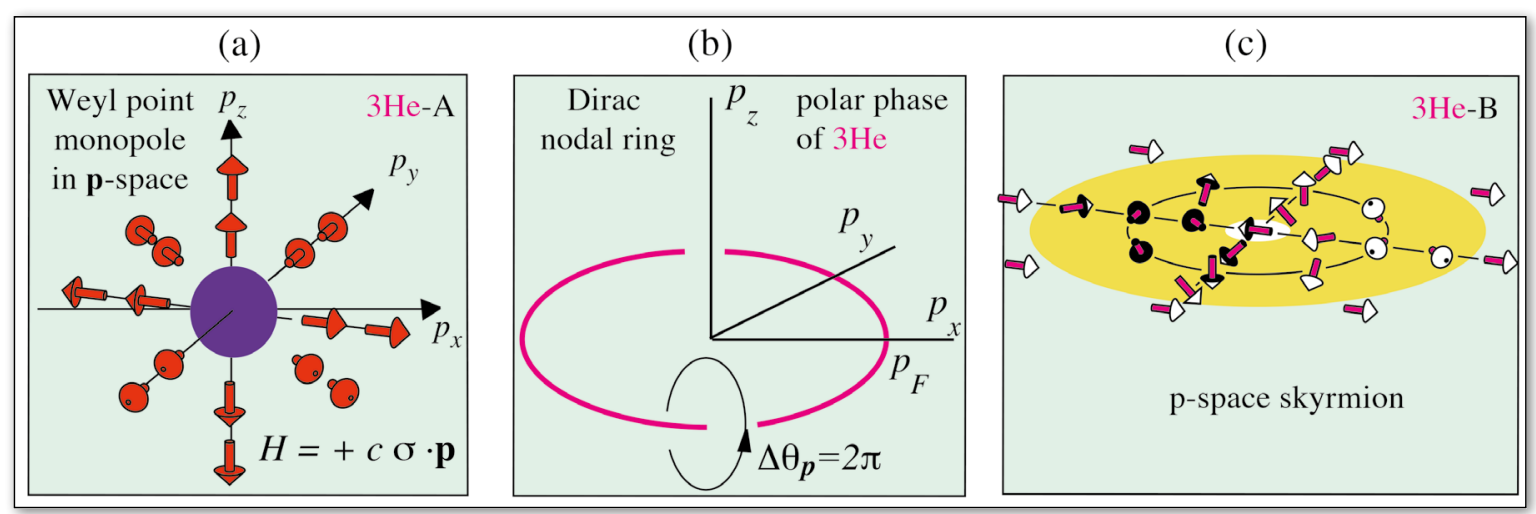




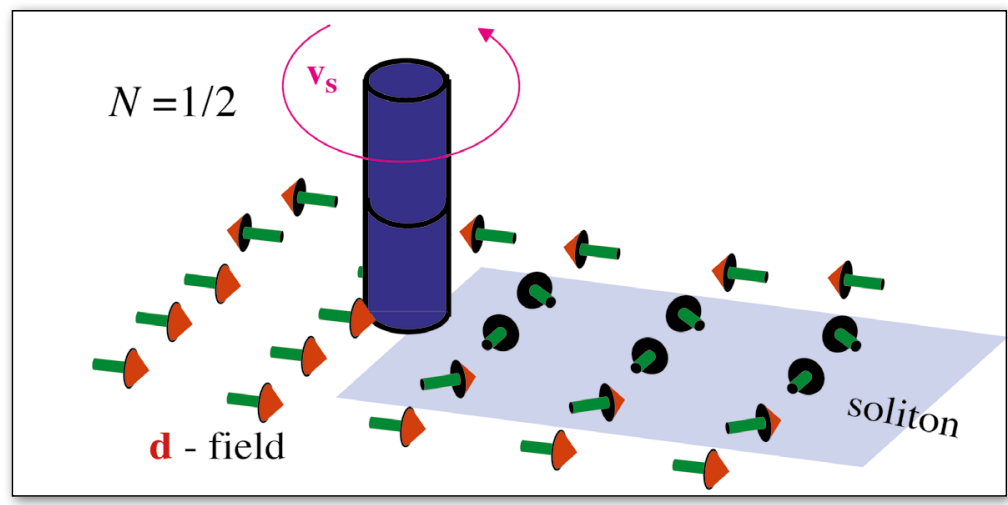

$\triangle$ FIG. 3: Half-quantum vortex in chiral superfluid ${ }^{3} \mathrm{He}$-A and in the polar phase. The spin part of the order parameter in both phases has the form $\Psi=\hat{\mathrm{d}} e^{i \phi}$. The phase $\Phi$ of this vector order parameter changes by $\pi$ around the half-quantum vortex. The change of the phase of the order parameter is compensated by the change of the direction of the spin vector $\hat{d}$ and do not produce physical jump in the order parameter $\psi$. The order parameter remains continuous around the vortex. $\ln ^{3} \mathrm{He}-\mathrm{A}$, the spin-orbit interaction forces the change of $\hat{\mathrm{d}}$-vector to be concentrated within the topological soliton. Across the soliton the $\hat{d}$ continuously changes the direction to the opposite. Due to the energy of the soliton attached to the vortex, the vortex is not favorable energetically. The half-quantum vortex has been observed first in the polar phase of ${ }^{3} \mathrm{He}$ confined in a nanostructured material called nafen [8]. In the polar phase the spin-orbit interaction is more favorable: in zero magneticfield or in the field along the nafen strands the solitons are absent, and the lattice of half quantum vortices becomes the lowest energy state in a rotating vessel.

- FIG.4: Half-quantum vortex as the Alice string. Spacetime continuously transforms to its mirror image after circling an Alice string, and thus matter continuously transforms to antimatter. Two penguins, Alice and Bob, start to move in opposite directions around the string.

When they meet each other again, they may annihilate. In condensed matter the vector $\hat{d}$, which is the axis of quantization for spin, changes sign around the half-quantum vortex, i.e. spin transforms to "antispin" - the analog of antimatter. a magnetic monopole in gauge theory, see Fig. 2 (a). Other condensed matter representatives of this class are solid state topological materials - Weyl semimetals and Weyl superconductors.

2) The polar phase has been realized in ${ }^{3} \mathrm{He}$ immersed in a nanostructured material called nafen, see e.g. Ref. [8]. Nafen is composed of nearly parallel solid strands, which are about $9 \mathrm{~nm}$ in diameter and are 30-50 nm apart. The nafen volume is thus mostly empty and in the experiments this empty space is filled by liquid ${ }^{3} \mathrm{He}$.

Quasiparticles in the polar phase are also gapless, but their energy is zero along a line in momentum space so-called Dirac nodal line in Fig. 2 (b). These quasiparticles are similar to massless quasi-two dimensional Dirac particles with one important reservation: the synthetic metric $g^{i k}$ becomes degenerate, i.e. the "speed of light" vanishes in the direction along the nodal line.

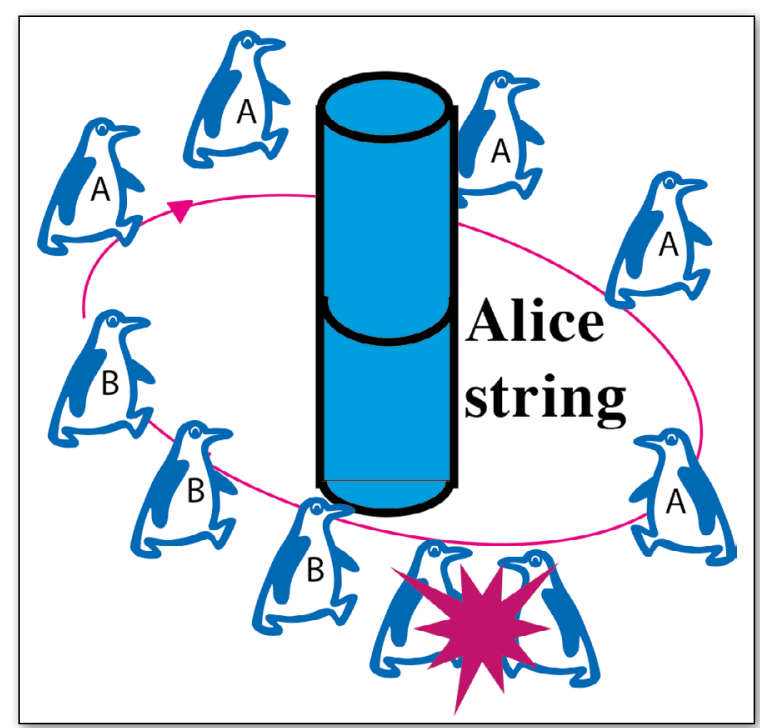

3 ) In the B phase $\left({ }^{3} \mathrm{He}-\mathrm{B}\right)$ the spectrum of quasiparticles is similar to the spectrum of Dirac particles in the present epoch of the Universe, where all elementary particles have became massive. The fully-gapped B-phase has the topology of the so-called DIII superconducting class, which is similar to the topology of skyrmions in real space Fig. 2 (c). This topological configuration protects massless Majorana fermions living on the surface of the superfluid and in vortex cores.

\section{Half-quantum vortex as an Alice string} One of the exotic topological objects living in ${ }^{3} \mathrm{He}$ is the half-quantum vortex (HQV) - a vortex encapsulating a fraction of the quantum of circulation, see Fig. 3. It is the analog of so-called Alice string in cosmology, where a particle encircling an Alice string continuously transforms to an antiparticle. In other words, the spacetime is continuously transformed to its mirror image - the antispacetime, see Fig. 4. In cosmology, antispacetime Universe was recently suggested as a continuation of our Universe across the Big Bang singularity [9]. This is a rather more dangerous route for Alice to travel to a mirror Universe - going around the Alice string can still be safe for Alice if she can avoid close encounter with Bob.

HQVs were originally predicted to exist in the chiral superfluid ${ }^{3} \mathrm{He}-\mathrm{A}[10]$. However, before being experimentally observed in ${ }^{3} \mathrm{He}-\mathrm{A}$, the HQVs were first observed in another topological phase of ${ }^{3} \mathrm{He}-$ the polar phase [11]. The reason for that is that in ${ }^{3} \mathrm{He}-\mathrm{A}$, the spin-orbit interaction chooses the preferable orientation for the vector $\hat{d}$ describing the spin degrees of freedom of the order parameter. This leads to formation of a soliton interpolating between two degenerate vacua in Fig. 3 . The energy of the soliton prevents the nucleation of the HQVs (Alice strings) in ${ }^{3} \mathrm{He}-\mathrm{A}$. In contrast, in the polar phase the spin-orbit interaction can be controlled to not prohibit the formation of HQVs.

The surprise was HQVs, which are formed in the polar phase by rotation of the superfluid or by the Kibble-Zurek mechanism, surviving the transition to the ${ }^{3} \mathrm{He}-\mathrm{A}$. The reason for that is that the defects are pinned by the nafen strands. They remain pinned after transition to the ${ }^{3} \mathrm{He}-\mathrm{A}$, in spite of the formation of the energetically costly solitons and therefore effective attractive tension between vortices.

\section{Two routes to mirror Universe}

Even more surprisingly, further experiments demonstrated that the HQVs survive even the phase transition to ${ }^{3} \mathrm{He}-$ $B$, where such defects cannot exist as individual entities. It was found that the HQV becomes part of a composite defect: it is the boundary of a domain wall in Fig. 5. As distinct from the continuous topological soliton in Fig. 3, the wall bounded by HQV is singular: it is composed of yet a different unstable superfluid phase with a degenerate 
metric, and has a higher energy. But still the wall tension is not sufficiently strong to unpin the vortices.

In cosmology, the walls bounded by cosmic strings have been suggested by Kibble, Lazarides and Shafi (KLS) [12]. The KLS walls appear after two successive cosmological phase transitions. Below the first transition the topologically stable defects - cosmic strings - are formed. Below the second transition these defects lose the topological stability and become boundaries of domain walls. As we explained, the same mechanism with successive phase transitions works in superfluid ${ }^{3} \mathrm{He}$. The composite HQV defect the Kibble-Lazarides-Shafi wall bounded by HQVs (Alice strings) - demonstrates the two ways to enter the mirror world in Fig. 6 [13]. The safe (continuous) route is around the half-quantum vortex and the dangerous route is across the cosmic singularity of the Kibble-Lazarides-Shafi wall. In our case the dangerous route is similar to the route of our Universe from spacetime to antispacetime in Ref. [9].

\section{Acknowledgements}

This work has been supported by the European Research Council (ERC) under the European Union's Horizon 2020 research and innovation programme (Grant Agreement No. 694248).

\section{About the Authors}

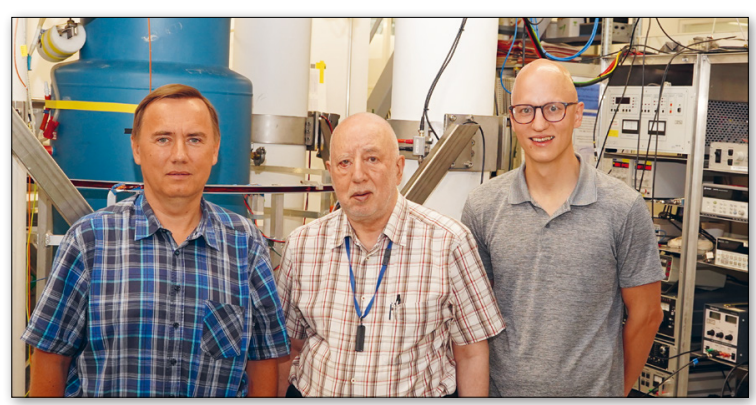

Grigory Volovik (middle) currently shares the position in Landau Institute (Chernogolovka, Russia) with a position in Aalto University (Otaniemi, Finland). The areas of his interests are topological defects; topological matter; connections between condensed matter physics, particle physics andcosmology. In 2003-2006 he was chairman of the European Science Foundation Programme "Cosmology in the Laboratory". He was awarded Landau Prize (1992), Simon Prize (2004) and Lars Onsager prize (2014); member of German National Academy Leopoldina.

Vladimir Eltsov(left) received his doctoral degree from the Kapitza Institute in Moscow, Russia in 1997 and then moved to Helsinki University of Technology to work on superfluid ${ }^{3} \mathrm{He}$. Since 2010 he leads the experimental research on rotating ${ }^{3} \mathrm{He}$ at Aalto University.

Jaakko Nissinen (right) is a post-doctoral research at Aalto University. His research interests include topological phases of matter, condensed matter analogs of relativistic quantum field theory and quantum Hall physics. He obtained his Ph.D. at the University of Oslo, Norway.

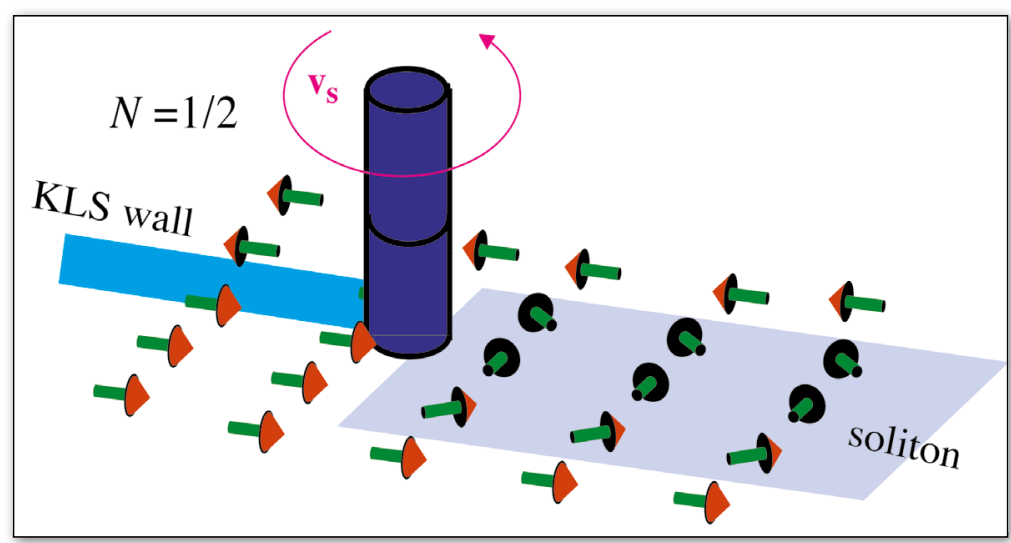

$\triangle$ FIG. 5: Further experiments demonstrated that the half-quantum vortex survives even the phase transition to ${ }^{3} \mathrm{He}-\mathrm{B}$, where such a vortex cannot exist as an independent topological object. The previously unobservable jump in the $\hat{d}$ field becomes physical — the domain wall between different superfluid vacua. This wall is singular as distinct from the continuous topological soliton. So, in ${ }^{3} \mathrm{He}-\mathrm{B}$ the half-quantum vortex becomes the part of the composite defect - the domain wall terminated by the string. This is the analog of the Kibble-Lazarides-Shafi (KLS) cosmic wall bounded by cosmic string [12].

\section{References}

[1] T.W.B. Kibble, J. Phys. A9, 1387 (1976).

[2] W. H. Zurek, Nature 317, 505 (1985).

[3] A.M. Polyakov, JETP Lett. 20, 194 (1974).

[4] G. 't Hooft, Nucl. Phys. 79, 276 (1974).

[5] V.M.H. Ruutu, V.B. Eltsov, A.J. Gill, T.W.B. Kibble, M. Krusius, Yu.G. Makhlin, B. Placais, G.E. Volovik, Wen Xu, Nature 382, 334 (1996).

[6] G.E. Volovik, The Universe in a Helium Droplet, Clarendon Press, Oxford (2003).

[7] T.D.C. Bevan, A.J. Manninen, J.B. Cook, J.R. Hook, H.E. Hall, T. Vachaspati and G.E. Volovik, Nature 386, 689 (1997).

[8] S. Autti, V.V. Dmitriev, J.T. Mäkinen, A.A. Soldatov, G.E. Volovik, A.N. Yudin, V.V. Zavjalov, and V.B. Eltsov, Phys. Rev. Lett. 117, 255301 (2016).

[9] L. Boyle, K. Finn and N. Turok, Phys. Rev. Lett. 121, 251301 (2018).

[10] G.E. Volovik, V.P. Mineev, JETP Lett. 24, 561 (1976).

[11] J.T. Mäkinen, V.V. Dmitriev, J. Nissinen, J. Rysti, G.E. Volovik, A.N. Yudin, K. Zhang, V.B. Eltsov, Nat. Comm. 10, 237 (2019).

[12] T.W.B. Kibble, G. Lazarides and Q. Shafi, Phys. Rev. D 26, 435 (1982).

[13] G.E. Volovik, JETP Lett. 109, 499 (2019).

VFIG. 6: Two roads to antispacetime: the safe route around the Alice string (along the contour $C_{1}$ ) or the dangerous route along $C_{2}$ across the Kibble-LazaridesShafi wall with degenerate metric. This dangerous route through the Alice looking glass is similar to the route of our Universe from spacetime to antispacetime via Big Bang in Ref. [9].

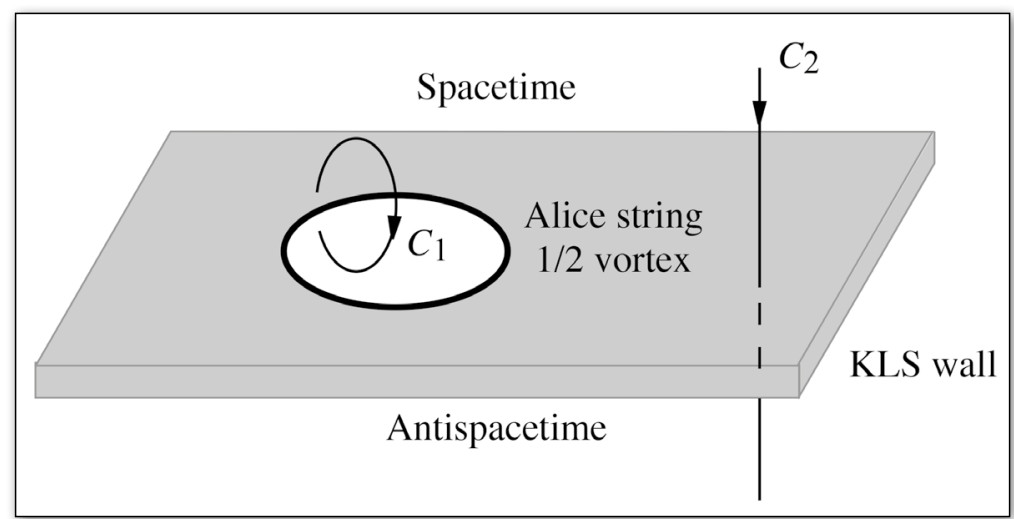

